\title{
Opponens Pollicis Muscle
}

National Cancer Institute

\section{Source}

National Cancer Institute. Opponens Pollicis Muscle. NCI Thesaurus. Code C150853.

A muscle that originates on the distal border of the flexor retinaculum and the tubercles

of the scaphoid and trapezium, and inserts into the lateral aspect of the first metacarpal;

it is involved in the rotation and flexion of the thumb and is supplied by the recurrent

branch of the medial nerve and the superficial palmar branch of the radial artery. 